\title{
Increased Permeability of Cutaneous Lymphatic Capillaries and Enhanced Blood Flow in Psoriatic Plaques
}

\author{
Thomas O. Meier $^{\mathrm{a}}$ Ludmila Kovacicova $^{\mathrm{a}}$ Reto Huggenberger ${ }^{\mathrm{c}}$ \\ Alexander A. Navarini ${ }^{b}$ Gabriela Gitzelmann ${ }^{a}$ Beatrice R. Amann-Vesti ${ }^{a}$ \\ ${ }^{a}$ Clinic for Angiology and b Department of Dermatology, University Hospital Zurich, and ${ }^{\mathrm{C} I n s t i t u t e}$ of Pharmaceutical \\ Sciences, Swiss Federal Institute of Technology, ETH Zurich, Zurich, Switzerland
}

\section{Key Words}

Psoriasis · Microcirculation · Lymph vessel · Laser Doppler flow · Fluorescence microlymphography

\begin{abstract}
Introduction: Morphological abnormalities of microvessels are described in psoriasis. However, there are conflicting data as to whether their function is also altered. Objective: Our aim was to study the morphology and function of the lymphatic capillaries of psoriatic skin. Methods: Morphology and permeability of initial lymphatics were studied by microlymphography and densitometry in 20 patients. Perfusion was studied by laser Doppler fluxmetry. Results: Permeability of lymphatics in plaques was increased by $7.6 \% \mathrm{com}$ pared to unafflicted skin $(p<0.001)$. Lymphatic vessel density and the extension of dye in lymphatic networks were not significantly different between involved and uninvolved areas. Both sites showed a wide range of diameters of lymphatics. The median laser Doppler flux in plaques was increased by $144 \%$ (91-380\%) compared to unaffected skin $(p<0.001)$. Conclusions: Increased permeability of lymphatics and increased blood flow was demonstrated in vivo in psoriatic skin lesions. These findings may reflect the local inflammatory process and may be used as markers when studying new therapeutic approaches for psoriasis.
\end{abstract}

(c) 2013 S. Karger AG, Basel

\section{KARGER}

(๑) 2013 S. Karger AG, Basel

$1018-8665 / 13 / 2272-0118 \$ 38.00 / 0$

E-Mail karger@karger.com

www.karger.com/drm

\section{Introduction}

Morphological abnormalities of blood capillaries and lymphatic capillaries are described in psoriasis. It is suggested that they play a fundamental role in the development of psoriatic plaques [1-3].

Histological and immunohistological as well as electron microscopic studies revealed tortuous and expanded skin capillaries with an increased lymphatic vessel density in psoriatic plaques $[1,4,5]$. Following the treatment of psoriasis a reduced number of lymphatic capillaries of the skin were reported [3]. Previous electron microscopic studies and skin biopsies of plaques demonstrated numerous abnormal dilated lymphatic capillaries within the lesions and less prominent ones in the uninvolved skin of psoriatic patients $[5,6]$. Furthermore, an increased number, width and length of the blood microvessels were found in skin biopsies of the plaques [7].

Currently, it is not clear whether there is only an anatomic abnormality of microvessels in psoriatic plaques or whether this also translates into a functional alteration with impaired drainage of lymphatic fluid and reduced perfusion.

To our knowledge, there are only three studies investigating the lymphatic drainage in psoriatic skin. All show an increased drainage $[3,8,9]$.

Thomas O. Meier, MD

Clinic for Angiology, University Hospital Zurich

Raemistrasse 100

CH-8091 Zurich (Switzerland)

E-Mail thomas.meier@usz.ch 
Laser Doppler fluxmetry with laser Doppler perfusion imaging (LDPI) or monitoring is a noninvasive technique to demonstrate skin perfusion. In several studies an increased laser Doppler flux in the plaques has been demonstrated [10-12].

Although the exact pathogenetic mechanisms leading to the microvascular abnormalities in psoriasis are unknown, there are indications that vascular endothelial growth factor-A (VEGF-A) plays a central role. It could be shown that transgenic mice that overexpress VEGF-A in the epidermis underwent a lymphatic vessel proliferation and enlargement and an increased and prolonged inflammatory response with skin changes resembling human psoriasis [6]. Systemic inhibition of VEGF-A or its receptors reduced the skin inflammation and also decreased lymphatic vessel size in different mouse models of psoriasis $[13,14]$. Interestingly, there is increasing evidence that blocking lymphangiogenesis prolongs the course of inflammation, as seen in mouse models of chronic airway inflammation [15], chronic rheumatoid arthritis [16] and chronic skin inflammation [14]. In contrast, stimulation of lymphangiogenesis and lymphatic function reduced chronic skin inflammation and chronic rheumatoid arthritis $[14,17]$.

Thus, inhibition of VEGF-A bioactivity and stimulation of lymphatic function might be a new therapeutic approach for psoriasis. Therefore, the aim of this study was to further characterize the function and morphology of lymphatic vessels in psoriatic plaques.

\section{Patients and Methods}

Patients

In this prospective study, outpatients suffering from moderate psoriasis vulgaris (plaque-type psoriasis) were included from June 2010 to April 2011. All patients had active psoriatic plaques and were treated either with phototherapy and topical agents or with systemic therapy.

In all patients the morphology and function of the lymphatic capillaries in the psoriatic plaques were studied by fluorescence microlymphography; the blood perfusion of the psoriatic skin lesion was examined by LDPI.

The study was approved by the local ethical committee (EKStV-Nr 2010/00144/5) and all patients gave written informed consent.

\section{Fluorescence Microlymphography}

The technique of fluorescence microlymphography has been described in detail [18]. Using a steel microcannula with a tip diameter of $0.2 \mathrm{~mm}$ (Arnold Bott, Zurich, Switzerland) connected to a microsyringe (Hamilton, Bonaduz, Switzerland), $10 \mu \mathrm{l}$ of a sterilized $25 \%$ solution of FITC-(fluorescein isothiocyanate) dex- tran 150,000 MW (TdB Consultancy AB, Uppsala, Sweden) was injected into the subepidermal layer of the skin in the middle of the psoriatic plaques. In large plaques a second injection at the border was performed to visualize lymphatics crossing from healthy into diseased skin. As large molecules are exclusively drained by the lymphatic system, the fluorescent dextran molecules move into the initial lymphatics and can be visualized by a fluorescence video microscopy system. This consists of an incident light fluorescence microscope (Leica, Heerbrugg, Switzerland), a 3-charge-coupled device video camera (model DXC930P, Sony, Tokyo, Japan) with a camera adapter and sensitivity on automatic control (CMA-D2, Sony), a timer (VTG-22) and scale marker (IV-600; both from For-A-Company, Tokyo, Japan), a monitor (Picture Monitor model PM171T, Ikegami Tsushinki, Tokyo, Japan) and a personal computer with a recorder software (synedra View and Image View software; synedra Information Technologies, Innsbruck, Austria). The microscope was equipped with $1.0 / 0.04,2.5 / 0.08,6.3 / 0.20$ and $10 / 0.25$ planar objectives (Leica), which allow a magnification of $\times 24, \times 62, \times 165$ and $\times 240$, respectively, on the monitor. The fluorescence excitation filter works at $450-490 \mathrm{~nm}$ and the barrier filter at $515 \mathrm{~nm}$.

\section{Morphology of Lymphatic Vessels}

The following parameters have been evaluated offline from the recordings $10 \mathrm{~min}$ after dye injection: presence and density of visualized lymphatic meshes, fragmentation of the superficial lymphatic network (defined as network with interruptions of the meshes), cutaneous backflow (defined as abnormal retrograde flow from deep to cutaneous lymphatics away from the main network), and maximal extension of the fluorescent macromolecules into the lymphatic network in the lateral, proximal, medial and distal direction as a measure for lymphatic drainage capacity into deeper channels and diameter of single lymphatic microvessels.

\section{Lymphatic Vessel Density}

Because it is difficult to count the number of vessels in a network, we used the total length of the lymphatic capillaries in a square millimeter as an equivalent to the lymphatic vessel density. The measurements were taken offline from the recordings $10 \mathrm{~min}$ after dye injection. The mean value of three measurements per recording was calculated. The $1-\mathrm{mm}^{2}$ large region of interest was localized about $2 \mathrm{~mm}$ away from the dye depot and where the lymphatic network was delineated.

\section{Lymphatic Permeability}

The lymphatic wall is permeable to water and small solutes but less to larger molecules such as FITC-dextran 150,000 MW in healthy skin. High peaks of fluorescent light intensity remain located over the capillary. Increased permeability is characterized by increased interstitial fluorescence intensity and a lower peak of light intensity over the vessel [19]. The permeability of initial lymphatics was determined by video densitometry. The recordings of the lymphatic network obtained during the microlymphography 10 min after dye injection were digitized and colors were transformed into black and white (synedra View 3 and Image View software; synedra Information Technologies). Single images of well-delineated lymphatic capillaries as far away as possible from 
the dye depot were chosen. A $10 \times 10$ pixel region of interest was localized over the brightest point in the lymphatic capillary and $0.5 \mathrm{~mm}$ away from the capillary wall in the interstitial tissue. The light intensity was measured 3 times by the image processing and analysis software ImageJ (a free available Java-based public-domain image processing and analysis program developed at the $\mathrm{Na}$ tional Institutes of Health by Wayne Rasband of the National Institute of Mental Health) and the median value was calculated. Light intensity was expressed in arbitrary density units and the light intensity in the vessel defined as $100 \%$. Permeability of cutaneous lymphatic vessels was calculated as follows: (light intensity in the vessel) $/ 100 \% \times($ light intensity in the interstitium).

\section{Laser Doppler Perfusion Imaging}

LDPI is a more recent method to determine the local distribution of flux over a skin area without direct contact with the tissue surface. The laser light was emitted from the source placed approximately $20 \mathrm{~cm}$ above the skin $[20,21]$. Flux is a relative measure of the microvascular flow, represents the product of speed and number of cells and is expressed in arbitrary perfusion units. The LDPI provides color-coded 2D images of the blood flow through the investigated tissue. In the pictures the lowest perfusion is represented by a dark blue color; when perfusion increases the colors change from light blue, green, yellow and orange to red. These images were analyzed with the LDPIWin system software (Perimed $\mathrm{AB}$, Stockholm, Sweden). The same region of interest in the subsequent two LDPI images of a certain skin area was selected and the median flux was calculated. The flux of the normal skin was defined as $100 \%$. The region of interest was the whole area of a psoriatic plaque.

The LDPI has an average measurement depth in human skin of about $1-1.5 \mathrm{~mm}$ and therefore the nutritive and thermoregulative microcirculation is examined $[21,22]$. In this study a PIM 2.0 laser Doppler perfusion imager (PeriScan PIM II, Perimed AB) was used, with a low-power helium-neon laser beam $(1 \mathrm{~mW}$, $670 \mathrm{~nm})$.

\section{Investigational Protocol}

All measurements were performed in a psoriatic plaque at one of the extremities or at the upper body when no plaque was present at the extremity. The same measurements were performed in the unaffected skin close to the plaque. During the procedure patients were in supine position with the leg at heart level. The temperature of the air-conditioned examination room was maintained between 22 and $26^{\circ} \mathrm{C}$ as recommended [23].

\section{Statistical Analysis}

Statistical analysis was performed using the statistical program IBM SPSS Statistics 20 (SPSS Inc., Chicago, Ill., USA). Results are presented as median and range. $\mathrm{p}$ values for nonnormal distributed results were calculated by Wilcoxon signed ranks test and for normal distributed results by the paired samples t test. Interplaque and intercapillary reliabilities were calculated as intraclass correlations (ICC) using restricted maximum likelihood estimators of variance components in a one-way model.

\section{Results}

\section{Patients}

Twenty psoriatic lesions of 20 patients with psoriasis vulgaris (4 female, median age 50 years, range 29-79) were studied. The mean duration of the disease was 16 years (range 1-55). The patients were examined when the psoriasis was in an active but stable phase; 1 patient had no treatment because the psoriasis had been recently diagnosed, 11 patients received a systemic treatment (methotrexate $n=6$, biologics $n=4$, PUVA $n=1$, narrow band $\mathrm{UVB} \mathrm{n}=2$ ) and 14 patients received a topical treatment (table 1).

\section{Fluorescence Microlymphography}

In 3 patients the assessment of the skin lymphatics was not possible due to impaired visibility of lymphatic vessels.

Diameters of 2-10 capillaries were measured per patient and lymphatic network. Because of the large variability of diameters (ICC 0.52 within plaques and 0.36 in uninvolved skin), medians of corresponding measures were analyzed. These diameters were significantly smaller in the plaques (median $44 \mu \mathrm{m}$, range $31-85$ ) than in the unafflicted skin (median $62 \mu \mathrm{m}$, range $47-110, \mathrm{p}=0.001$ ) (fig. 1, 2). Extensions of two plaques were measured per patient. They had a high reliability (ICC 0.75 of single measures) and hence the mean of these two measures was analyzed. No reliability for extensions in unaffected skin could be calculated. The median extension of the dye was equal between plaques $(3.0 \mathrm{~mm}$, range $1-12)$ and perilesional skin (2.5 mm, range $1-16, \mathrm{p}=0.357)$ (fig. 3). Importantly, the permeability of the lymphatics in the plaques was significantly increased by $7.6 \%$ (95\% confidence interval, CI, 10.4-4.8) from $73.2 \%$ (range 64.7-89.7) in the uninvolved skin to $81.6 \%$ (range $70.2-92.8$ ) in the plaque $(\mathrm{p}<0.001)$ (fig. 4). The density of the depicted lymphatic vessels, expressed as the total length of the lymphatic network in a square millimeter, was very variable and not significantly increased in the plaques. One to three lymphatic networks per patient were measured. The mean value in the plaques was $2,773 \mu \mathrm{m}$ (range 1,033-6,931) and in the uninvolved skin $2,162 \mu \mathrm{m}$ (range 659-6,180, $\mathrm{p}=0.084$ ) (fig. 5). Neither fragmentation of the superficial lymphatic network nor cutaneous backflow were observed.

\section{Laser Doppler Perfusion Imaging}

The flux of one plaque per patient was measured. The median laser Doppler flux in plaques was significantly increased to $144 \%$ (range $91-380$ ) of the flux in the uninvolved skin $(\mathrm{p}<0.001)$ (fig. 6). 


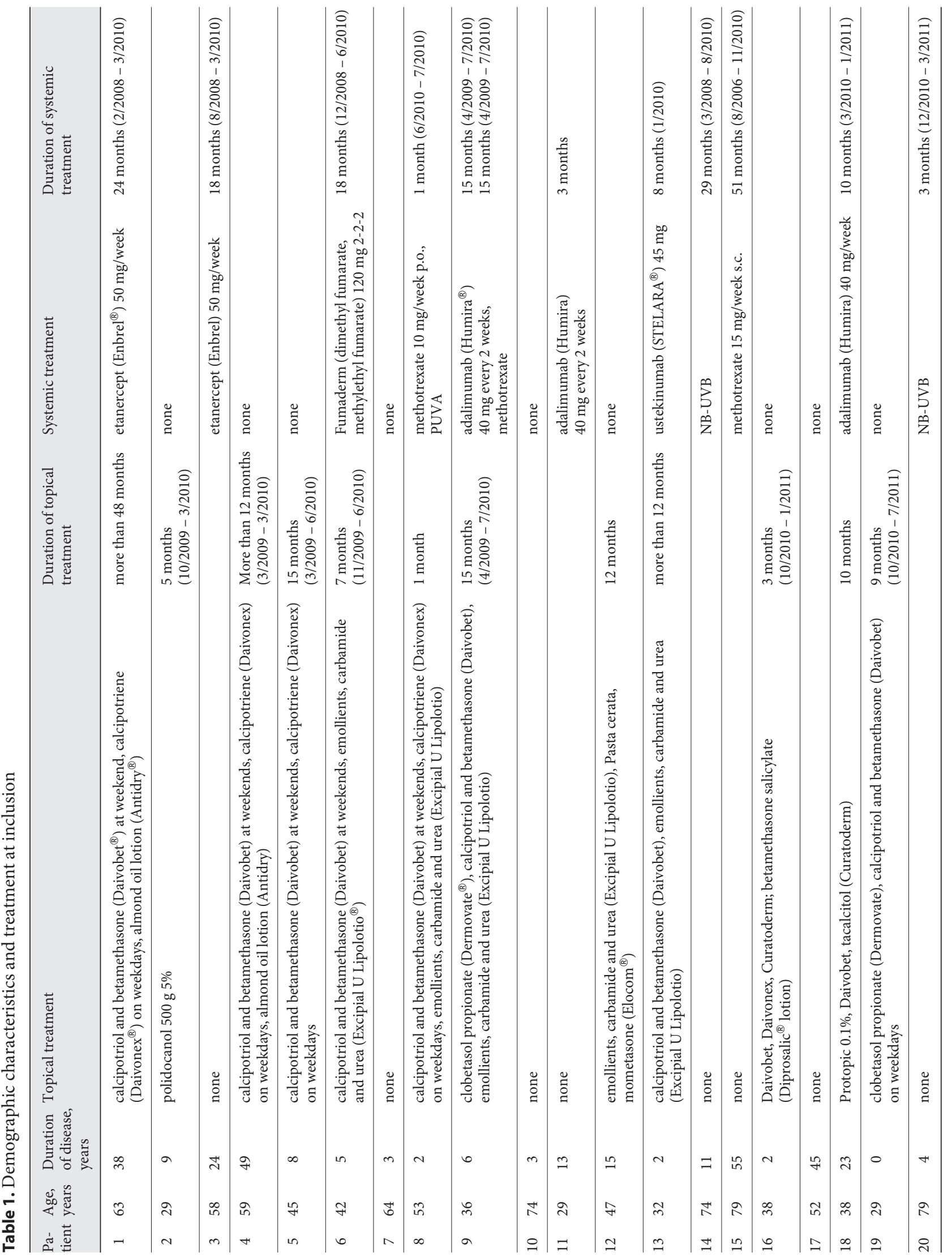



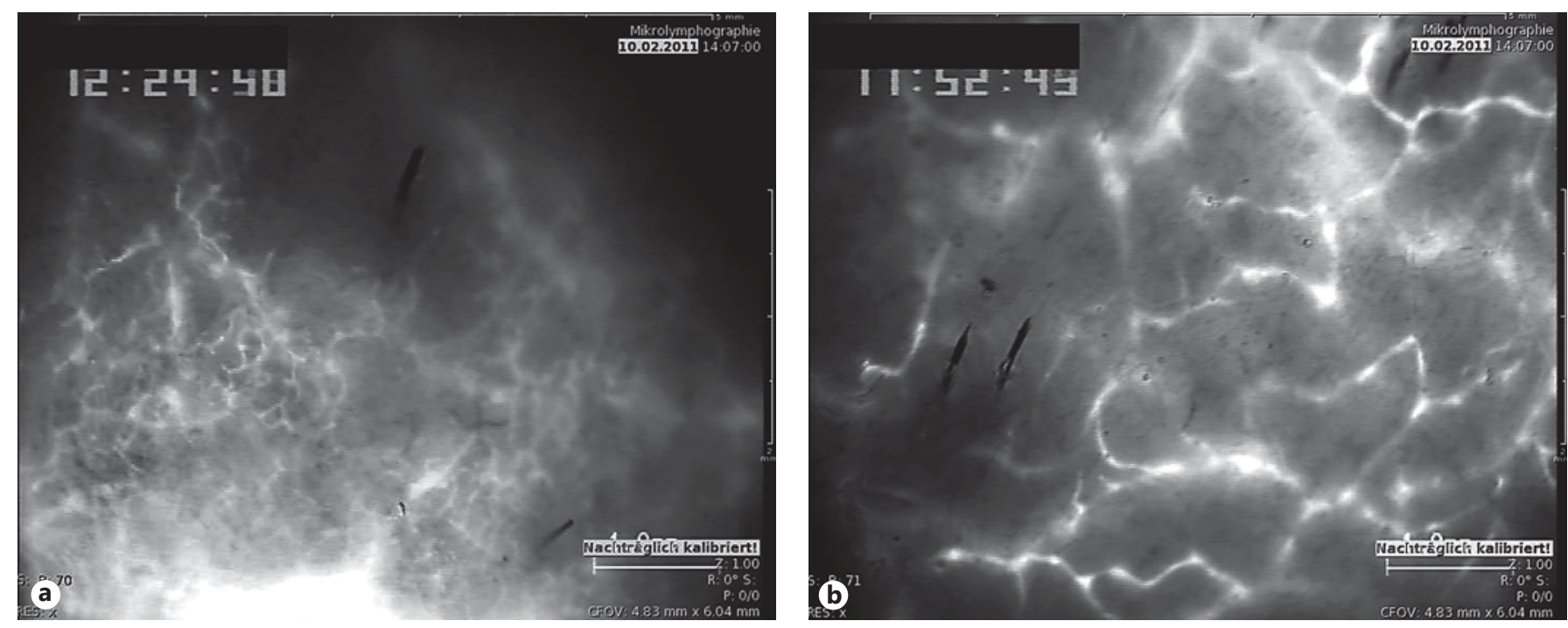

Fig. 1. Fluorescence microlymphography in the skin of a patient with psoriasis shows the network of smaller lymphatics in the plaque (a) than in the perilesional skin (b). Magnification $\times 24$.

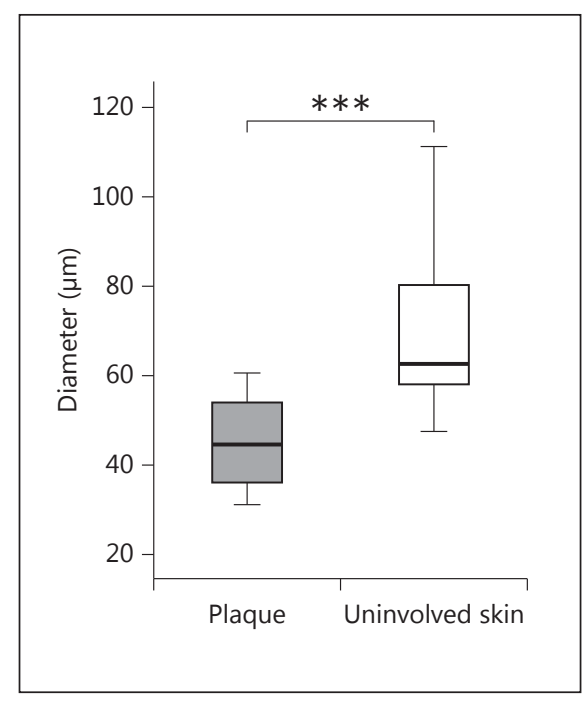

Fig. 2. The median diameter of lymphatic capillaries in the plaques is significantly smaller than in the perilesional skin. The number of measurements in plaque was 111 and in uninvolved skin it was 99. $* * * \mathrm{p}=0.001$.

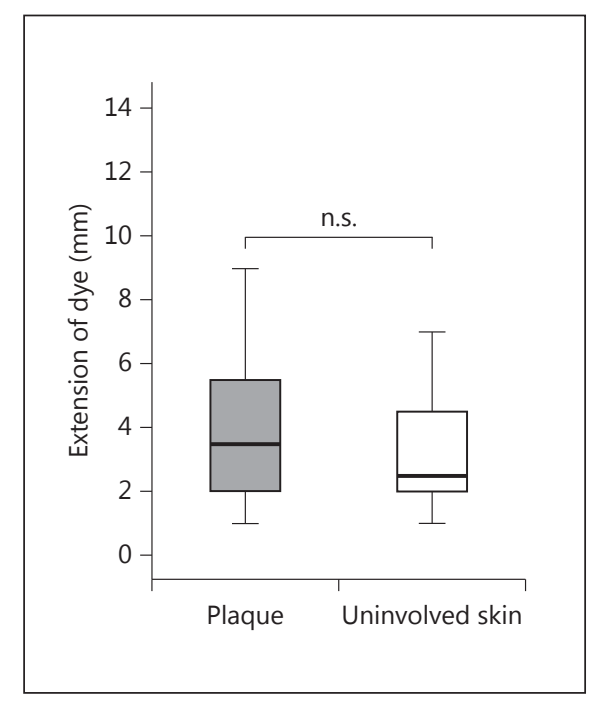

Fig. 3. The median extension of dye in lymphatic networks of plaques and perilesional skin was not significantly different. The number of measurements in plaque was 38 and in uninvolved skin it was 22. n.s. = Not significant.

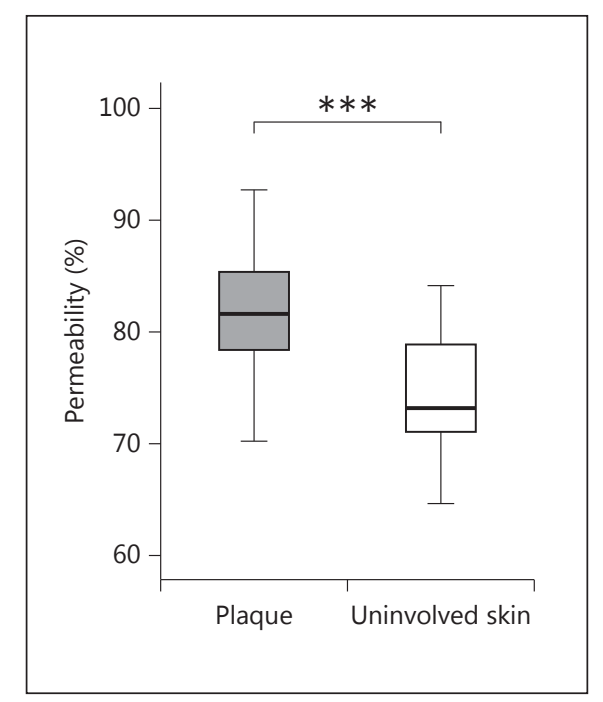

Fig. 4. The permeability of the lymphatics in the psoriatic plaques was significantly increased. The number of measurements in plaque was 40 and in uninvolved skin it was 40. ${ }^{* * *} \mathrm{p}<0.001$.

\section{Discussion}

The two main findings of this study were that the lymphatic capillaries in psoriatic skin are very variable in size and density, even more in the perilesional skin than with- in the psoriatic lesions itself, and that their permeability is increased in the plaque.

The diameter of the lymphatics was significantly smaller in the plaques (with a median diameter of $44 \mu \mathrm{m}$ ) compared to the perilesional skin (with a median diam- 
5

Fig. 5. The lymphatic vessel density in psoriatic plaques was not significantly increased. The number of measurements in plaque was 54 and in uninvolved skin it was 54. n.s. = Not significant.

Fig. 6. The median flux in the psoriatic skin was significantly higher than in the perilesional skin $(p<0.001)$. The number of measurements in plaque and in uninvolved skin was $20 . \mathrm{PU}=$ Perfusion units.
6

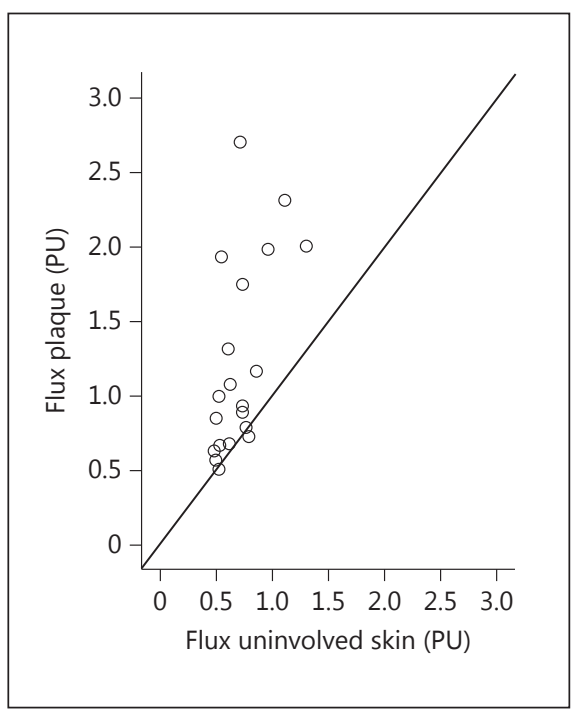

eter of $62 \mu \mathrm{m}$ that corresponds to the diameter of normal nonpsoriatic skin) [24]. These small lymphatic capillaries seem to be proliferating vessels probably in response to the treatment of the inflammatory process. Our in vivo findings are in contrast to previous micromorphological results of electron microscopic and immunohistological investigations demonstrating numerous dilated lymphatic capillaries in the skin of psoriatic patients $[5,6]$. In most of these studies the treatment was interrupted prior to the examination. The electron microscopic studies by Braverman and Yen [5] in the psoriatic skin also showed the presence of isolated blind lymphatic channels between the interconnecting lymphatic capillaries. The authors hypothesized that these channels might represent a lymphatic reserve left by previous inflammatory episodes that is capable of proliferating and connecting with the existing channels in response to inflammation or edema [5]. The classification of lymphatic skin vessels by diameter $(<10,10-17,18-24$ and $>24 \mu \mathrm{m})$ proposed by Jackowski et al. [25] to show the distribution of the microvessel calibers during different stages after radiotherapy of tumors was not applicable to our in vivo results in psoriatic patients since these lymphatics were larger than $24 \mu \mathrm{m}$.

In the present study no significant difference of the lymphatic vessel density between the psoriatic lesions and the uninvolved skin was found. Cliff et al. [26] published microlymphographic results in 6 patients with an even lesser density in the psoriatic lesion than in the nonlesional skin. Obviously, the microlymphographic findings

Function and Morphology of Lymphatic Vessels in Psoriatic Plaques are at odds with the higher density in the plaques described in previous ultrastructural studies with electron microscopy, histological and immunohistochemical examinations. Histological studies report an increase of the lymphatic vessel density or a shift of lymphatic vessels from the upper dermal plexus in deeper parts of the dermis [1]. In their immunohistochemical studies Fiedler et al. [4] found a marked increase in D2-40-positive lymphatic vessel density above the upper blood plexus in psoriatic plaques compared to normal skin samples. Lymphatic vessel segments in the dermal papillae were found in psoriasis in $87.5 \%$ and in normal skin in $9.4 \%$ of the 32 samples. The authors explain the higher lymphatic density by a possible upregulation to meet the needs of a better lymphatic drainage of the increased interstitial fluid in the plaques. This could well explain that following the treatment of psoriasis the number of lymphatic capillaries is reduced corresponding to the reduced inflammation [3]. It is also known that the lymphatic capillaries vanish when the psoriatic plaque starts to resolve [27]. This may explain the lower density in our treated patients.

Furthermore, in our study the extension of dye in the superficial lymphatic network of the psoriatic skin was normal with median values less than $12 \mathrm{~mm}$, indicating a normal lymphatic drainage both in the lesional and the perilesional skin [18]. This normal lymphatic drainage in the plaques was also described by Cliff et al. [26]. It is known from the examination by Staberg et al. $[8,9]$ that the lymphatic albumin clearance is increased in psoriatic skin when compared with normal skin. The authors ex- 
plain the presence of edema in the plaque with an increased leakage of the blood capillaries. The increased lymphatic drainage might compensate the fluid accumulation.

The significantly increased permeability of the lymphatics by $7.5 \%$ indicates not only morphological but also functional changes of the lymphatics in psoriatic plaques. To our knowledge this is the first time that measurements of permeability of lymphatics in psoriatic skin has become published. Adequately measured lymphatic permeability is shown since it is not confounded by edema. On the other hand the size of the measured area was limited by the vessel diameter. Therefore, a $10 \times 10$ pixel region was chosen for the calculation of the permeability. The number of measurement points was limited by the interfering light intensity of neighboring vessels. The interand intraindividual representativeness could not be demonstrated.

The discrepancy between our results and the results published to date might be due to the different inflammatory state of the studied plaques. Moreover, the results vary widely with the method of detection. In the present in vivo study perfused microvessels and their function are presented. In all previous studies except one micromorphological findings of (immune) histology or electron microscopy are described. These methods also show the nonperfused microvessels and there is a tissue-shrinking artifact by fixation; both might influence the results.

Further studies using in vivo microlymphography of the psoriatic plaques at different time points with different therapeutic regiments will be performed to confirm this hypothesis. The results of these studies will be more reliable if they are conducted with patients having an active disease before therapy and then compared with patients after successful therapy or at least during therapy.

In the present study the arterial perfusion measured by laser Doppler flux was increased in the plaques compared to the perilesional skin. The higher flux within the psoriatic lesions was also mentioned in other studies [12]. It is also known that the locally and neutrally mediated vasoregulation is intact in psoriatic plaques [28-30]. Davison et al. [7] demonstrated that the flux is increased up to $4 \mathrm{~mm}$ away from the plaque, whereas no epidermal changes and no lymphocytic excess have been confirmed histologically at the comparable active edge. This may indicate that an increased blood flow occurs early in psoriatic lesions well before the histological changes are present. The underlying mechanism of the increased blood flow in the psoriatic plaques is not known. Psoriasis is an autoimmune disease with inflammation in the active plaques. The elevated blood flow might be an indicator of the cutaneous inflammation and vascularization in the plaques as well as an indicator of the expansion of the plaques [7, 31].

Therefore, laser Doppler fluxmetry might be a marker for monitoring the effect of treatment of psoriasis. There are some studies examining this topic but the results are not uniform: PUVA phototherapy may have an influence on the dermal microvascular blood flow in contrast to narrow-band UVB phototherapy and pulsed dye laser $[10,11,30,32]$. Alternatively, laser Doppler flux may not be a good surrogate marker of clinical response to narrow-band UVB or laser phototherapy in contrast to PUVA phototherapy.

\section{Conclusion}

In psoriatic skin lesions increased permeability of lymphatics and increased arterial skin perfusion are present. These in vivo findings may reflect the local inflammatory process. Both lymphatic permeability and laser Doppler flux might be good markers when studying new therapeutic options for psoriasis.

\section{Acknowledgment}

We are indebted to Burkhardt Seifert, $\mathrm{PhD}$, Institute for Social and Preventive Medicine, Biostatistics Unit, University of Zurich, Switzerland (burkhardt.seifert@ifspm.uzh.ch) who reviewed the manuscript as a statistician.

\section{Disclosure Statement}

The authors have no conflict of interest or financial support to disclose.

References

Meier/Kovacicova/Huggenberger/ Navarini/Gitzelmann/Amann-Vesti Altmeyer P: In psoriasis the epidermis, including the subepidermal vascular plexus, grows downwards into the dermis. Br J Dermatol 1997;136:97-101.

2 Bhushan M, Moore T, Herrick AL, Griffiths CE: Nailfold video capillaroscopy in psoriasis. Br J Dermatol 2000;142:1171-1176.

3 Braverman IM, Sibley J: Role of the microcirculation in the treatment and pathogenesis of psoriasis. J Invest Dermatol 1982;78:12-17.

44 Fiedler E, Helmbold P, Marsch WC: Increased vessel density in psoriasis: involvement of lymphatic vessels in the papillary dermis. Br J Dermatol 2008;159:258-261. 
-5 Braverman IM, Yen A: Microcirculation in psoriatic skin. J Invest Dermatol 1974;62:493-502.

-6 Kunstfeld R, Hirakawa S, Hong YK, Schacht V, Lange-Asschenfeldt B, Velasco P, Lin C, Fiebiger E, Wei X, Wu Y, Hicklin D, Bohlen P, Detmar M: Induction of cutaneous delayedtype hypersensitivity reactions in VEGF-A transgenic mice results in chronic skin inflammation associated with persistent lymphatic hyperplasia. Blood 2004;104:1048-1057.

7 Davison SC, Ballsdon A, Allen MH, Barker JN: Early migration of cutaneous lymphocyte-associated antigen (CLA) positive t cells into evolving psoriatic plaques. Exp Dermatol 2001; 10:280-285.

8 Staberg B: Psoriasis. Transcapillary and interstitial transport of plasma proteins, cutaneous blood, flow, and effect of phototherapy. Dan Med Bull 1985;32:295-308.

-9 Staberg B, Klemp P, Aasted M, Worm AM, Lund P: Lymphatic albumin clearance from psoriatic skin. J Am Acad Dermatol 1983;9: 857-861.

10 Khan A, Schall LM, Tur E, Maibach HI, Guy $\mathrm{RH}$ : Blood flow in psoriatic skin lesions: the effect of treatment. Br J Dermatol 1987;117: 193-201.

11 Goh CL, Khoo L: Laser Doppler perfusion imaging (LDPI) and transepidermal water loss (TEWL) values in psoriatic lesions treated with narrow band UVB phototherapy. Dermal vascularity may be useful indicator of psoriatic activity. Ann Acad Med Singapore 2004;33:75-79.

$\checkmark 12$ Murray AK, Herrick AL, Moore TL, King TA, Griffiths CE: Dual wavelength (532 and 633 $\mathrm{nm}$ ) laser Doppler imaging of plaque psoriasis. Br J Dermatol 2005;152:1182-1186.

-13 Schonthaler HB, Huggenberger R, Wculek SK, Detmar M, Wagner EF: Systemic antiVEGF treatment strongly reduces skin inflammation in a mouse model of psoriasis. Proc Nat Acad Sci USA 2009;106:2126421269.
14 Huggenberger R, Ullmann S, Proulx ST, Pytowski B, Alitalo K, Detmar M: Stimulation of lymphangiogenesis via VEGFR-3 inhibits chronic skin inflammation. J Exp Med 2010; 207:2255-2269.

15 Baluk P, Tammela T, Ator E, Lyubynska N, Achen MG, Hicklin DJ, Jeltsch M, Petrova TV, Pytowski B, Stacker SA, Yla-Herttuala S, Jackson DG, Alitalo K, McDonald DM Pathogenesis of persistent lymphatic vessel hyperplasia in chronic airway inflammation. J Clin Invest 2005;115:247-257.

16 Guo R, Zhou Q, Proulx ST, Wood R, Ji RC, Ritchlin CT, Pytowski B, Zhu Z, Wang YJ, Schwarz EM, Xing L: Inhibition of lymphangiogenesis and lymphatic drainage via vascular endothelial growth factor receptor 3 blockade increases the severity of inflammation in a mouse model of chronic inflammatory arthritis. Arthritis Rheum 2009;60:2666-2676.

17 Zhou Q, Guo R, Wood R, Boyce BF, Liang Q, Wang YJ, Schwarz EM, Xing L: Vascular endothelial growth factor $\mathrm{C}$ attenuates joint damage in chronic inflammatory arthritis by accelerating local lymphatic drainage in mice. Arthritis Rheum 2011;63:2318-2328.

18 Bollinger A, Fagrell B: Clinical Capillaroscopy: A Guide to Its Use in Clinical Research and Practice. Toronto, Hogrefe \& Huber, 1990.

19 Huber M, Franzeck UK, Bollinger A: Permeability of superficial lymphatic capillaries in human skin to FITC-labelled dextrans 40,000 and 150,000. Int J Microcirc Clin Exp 1984;3: 59-69.

20 Kloppenberg FW, Beerthuizen GI, ten Duis HJ: Perfusion of burn wounds assessed by laser Doppler imaging is related to burn depth and healing time. Burns 2001;27:359-363.

21 Fullerton A, Stucker M, Wilhelm KP, Wardell K, Anderson C, Fischer T, Nilsson GE, Serup $\mathrm{J}$ : Guidelines for visualization of cutaneous blood flow by laser Doppler perfusion imaging. A report from the standardization group of the European Society of Contact Dermatitis based upon the HIRELADO European Community Project. Contact Dermatitis 2002;46: 129-140.
22 Murray AK, Gorodkin RE, Moore TL, Gush RJ, Herrick AL, King TA: Comparison of red and green laser Doppler imaging of blood flow. Lasers Surg Med 2004;35:191-200.

23 Pape SA, Skouras CA, Byrne PO: An audit of the use of laser Doppler imaging (LDI) in the assessment of burns of intermediate depth. Burns 2001;27:233-239.

24 Bollinger A, Jager K, Sgier F, Seglias J: Fluorescence microlymphography. Circulation 1981;64:1195-1200.

25 Jackowski S, Janusch M, Fiedler E, Marsch WC, Ulbrich EJ, Gaisbauer G, Dunst J, Kerjaschki D, Helmbold P: Radiogenic lymphangiogenesis in the skin. Am J Pathol 2007;171: 338-348.

26 Cliff S, Bedlow AJ, Stanton AW, Mortimer PS: An in vivo study of the microlymphatics in psoriasis using fluorescence microlymphography. Br J Dermatol 1999;140:61-66.

27 Braverman IM: The role of blood vessels and lymphatics in cutaneous inflammatory processes: an overview. Br J Dermatol 1983;109 (suppl 25):89-98.

28 Krogstad AL, Swanbeck G, Wallin BG: Axonreflex-mediated vasodilatation in the psoriatic plaque? J Invest Dermatol 1995; 104:872876.

29 Hern S, Stanton AW, Mellor R, Levick JR, Mortimer PS: Control of cutaneous blood vessels in psoriatic plaques. J Invest Dermatol 1999;113:127-132.

30 Hern S, Stanton AW, Mellor RH, Harland CC, Levick JR, Mortimer PS: Blood flow in psoriatic plaques before and after selective treatment of the superficial capillaries. $\mathrm{Br} \mathrm{J}$ Dermatol 2005;152:60-65.

31 Qin J, Jiang J, An L, Gareau D, Wang RK: In vivo volumetric imaging of microcirculation within human skin under psoriatic conditions using optical microangiography. Lasers Surg Med 2011;43:122-129.

- 32 Suh DH, Kwon TE, Kim SD, Park SB, Kwon OS, Eun HC, Youn JI: Changes of skin blood flow and color on lesional and control sites during PUVA therapy for psoriasis. J Am Acad Dermatol 2001;44:987-994. 